\title{
A Positive Touch: C-tactile afferent targeted skin stimulation carries an appetitive motivational value
}

Ralph Pawling ${ }^{\mathrm{a},+}$, Paula D. Trotter ${ }^{\mathrm{a}, \mathrm{b},+}$, Francis P. McGlone ${ }^{\mathrm{a}, \mathrm{c}}$ \& Susannah C. Walker ${ }^{\mathrm{a}^{*}}$

${ }^{a}$ Research Centre for Brain \& Behaviour,

School of Natural Sciences \& Psychology

Liverpool John Moores University,

Liverpool,

UK

${ }^{\mathrm{b}}$ Department of Psychology,

Manchester Metropolitan University,

Manchester,

UK

${ }^{\mathrm{c}}$ Institute of Psychology, Health \& Society,

University of Liverpool,

Liverpool,

UK

+ These authors contributed equally to this work

*corresponding author

Email: s.c.walker@1jmu.ac.uk

Tel: +44 (0) 1519046309 


\begin{abstract}
The rewarding sensation of touch in affiliative interactions is hypothesised to be underpinned by an unmyelinated system of nerve fibres called C-tactile afferents (CTs). CTs are velocity tuned, responding optimally to slow, gentle touch, typical of a caress. Here we used evaluative conditioning to examine whether $\mathrm{CT}$ activation carries a positive affective value. A set of neutral faces were paired with robotically delivered touch to the forearm. With half the faces touch was delivered at a CT optimal velocity of $3 \mathrm{~cm} / \mathrm{second}(\mathrm{CT}$ touch) and with the other half at a faster, non-CT optimal velocity of $30 \mathrm{~cm} / \mathrm{second}$ (Control touch). Heart-rate and skin conductance responses (SCRs) were recorded throughout. Whilst rated equally approachable pre-conditioning, postconditioning faces paired with CT touch were judged significantly more approachable than those paired with Control touch. CT touch also elicited significantly greater heartrate deceleration and lower amplitude SCRs than Control touch. The results indicate CT touch carries a positive affective value, which can be acquired by socially relevant stimuli it is associated with.
\end{abstract}

Keywords: Social; Touch; C-Tactile Afferent; Affective Touch, Evaluative Conditioning; ECG; SCR 


\section{Introduction}

Motivated reactions to emotionally salient stimuli can be broadly grouped into two categories, aversive and appetitive. While aversive reactions are observed in response to environmental stimuli or contexts that threaten survival, ultimately leading to withdrawal or escape, the appetitive motivational system is activated by stimuli which promote survival, signalling for example, food, sex or social interaction (Bradley, Codispoti, Sabatinelli, \& Lang, 2001; Lang \& Bradley, 2010). In a range of social species, across the life span, affiliative behaviours are associated with activation of the brain's reward systems and in sensory terms, touch is a key component of the rewarding value of such interactions (Dunbar, 2010; Loseth, Ellingsen, \& Leknes, 2014; Walker \& McGlone, 2013 for reviews).

C-Tactile afferents (CTs) are a class of unmyelinated low threshold mechanoafferent found in the hairy skin of mammals (see McGlone, Wessberg, \& Olausson, 2014 for recent overview). The specific response properties of these cutaneous afferents have been characterised using the electrophysiological technique microneurography. CTs respond optimally to a skin temperature stimulus moving across their receptive field at between 1-10cm/sec (Ackerley et al., 2014; Löken, Wessberg, Morrison, McGlone, \& Olausson, 2009; Vallbo, Olausson, \& Wessberg, 1999). This velocity tuning distinguishes them from larger diameter myelinated mechanoafferents, which respond linearly to stimuli of increasing velocity. In contrast, CTs show an inverted U shaped response function, responding less strongly to speeds that are either slower or faster than their preferred stimulus (Löken et al., 2009; Vallbo et al., 1999). Intriguingly, CT firing frequency is positively correlated with people's perceptions of stroking touch 
pleasantness. That is, the stimuli which CTs respond most strongly to are also those which are rated as subjectively most pleasant (Essick, James, \& McGlone, 1999; Essick et al., 2010; Löken et al., 2009). While CTs are found as frequently as other C-fibres coding pain and itch in the hairy skin of the body, in humans they have never been found in the glabrous skin of the palms of the hands or soles of the feet (McGlone, Wessberg, \& Olausson, 2014; Olausson, Wessberg, Morrison, McGlone, \& Vallbo, 2010).

The social touch hypothesis proposes that CTs' response characteristics and central projections make them ideally suited to signal the positive affective value of socially relevant tactile interactions (Morrison, Löken, \& Olausson, 2010; Olausson et al., 2010). Direct evidence that CT activating touch is rewarding comes from rodent studies where stroking the back of rats, at a CT optimal velocity, has been reported to induce dopamine release within the Nucleus Accumbens (Maruyama, Shimoju, Ohkubo, Maruyama, \& Kurosawa, 2012). Additionally, selective pharmacogenetic activation of C-low threshold mechanoreceptors (CLTMs - the animal homologue of CTs), which respond to massage like stroking, promotes the formation of conditioned place preference (Vrontou, Wong, Rau, Koerber, \& Anderson, 2013). In humans, psychophysical and fMRI investigations reliably show that CT optimal stimulation is preferred over slower and faster velocity stimulation (Essick et al., 1999; Löken et al., 2009; Perini, Morrison, \& Olausson, 2015) and leads to activation in reward related neural regions, such as the orbitofrontal cortex (Gordon et al., 2013; McGlone et al., 2012; Trotter et al., 2016). Recently, using facial EMG, we reported that CT optimal velocity touch on the forearm, but not the glabrous skin of the palm, elicited a 
significantly greater activation of the zygomaticus major (smile) muscle than did faster, non-CT optimal stroking (Pawling, Cannon, McGlone, \& Walker, 2017). Given enhanced activity over this muscle is associated with processing pleasant stimuli (Bradley et al., 2001; Cacioppo \& Tassinary, 1992; Larsen, Norris, \& Cacioppo, 2003), this finding provides further evidence of the positive affective value of $\mathrm{CT}$ activation in humans.

In the present study, we set out to further test the social touch hypothesis by determining whether touch, which optimally activates CTs, carries a positive affective value that can be acquired by previously neutral stimuli it becomes associated with. Evaluative conditioning refers to a change in the value of a neutral stimulus as a result of its repeated co-occurrence with a valenced stimulus (De Houwer, Thomas, \& Baeyens, 2001; Hofmann, De Houwer, Perugini, Baeyens, \& Crombez, 2010). Previous evaluative conditioning studies have reported reductions in explicit affective ratings and speeding of reaction times to neutral faces following repeated pairing with an unpleasant electrocutaneous stimulus (Andreatta \& Pauli, 2015; Bradley, 2009; Petrovic, Kalisch, Singer, \& Dolan, 2008). Here we paired the presentation of faces participants had previously rated as moderately approachable with touch delivered by a robotic tactile stimulator that was either $\mathrm{CT}$ optimal in terms of velocity $(3 \mathrm{~cm} / \mathrm{sec}-\mathrm{CT}$ touch) or Non-CT optimal (30 $\mathrm{cm} / \mathrm{sec}-$ Control Touch). Our primary hypothesis was that, if CT touch carries a positive affective value, post conditioning we would see a significant increase in the explicit approachability ratings of CT-touch paired faces compared to those paired with Control touch. Secondly, given that positive emotional stimuli have been shown, like aversive stimuli, to capture attention, we included a dot 
probe task as an additional, implicit measure of conditioning (Pool, Brosch, Delplanque, \& Sander, 2016). Our hypothesis was that reaction times to faces paired with CT touch, having acquired a positive affective value, would elicit significantly faster reaction times than those paired with Control touch.

Several previous studies have reported that social touch leads to a general increase in parasympathetic nervous system activity (Ditzen et al., 2007; Grewen, Girdler, Amico, \& Light, 2005; Light, Grewen, \& Amico, 2005). More specifically, CT optimal velocity touch has been reported to decrease the heart rate of both adults and infants to a significantly greater degree than faster or slower velocity stroking (Fairhurst, Löken, \& Grossmann, 2014; Pawling et al 2017). Additionally, in both healthy controls and two rare neuronopathy patients who have lost all large myelinated afferents, CT targeted brush stokes have been shown to elicit a sympathetic response (Olausson et al., 2008). Physiologically, exposure to emotionally salient stimuli, either appetitive or aversive, initially leads to co-activation of the parasympathetic and sympathetic branches of the autonomic nervous system (Bradley et al., 2001; Lang \& Bradley, 2010). Functionally, these reflex reactions capture attention, prioritising perceptual processing and prepare the body for action (approach or avoidance) (Bradley, Keil, \& Lang, 2012; Löw et al., 2008). Thus, during conditioning we collected both heart rate and skin conductance responses (SCR), measures of parasympathetic and sympathetic nervous system activity respectively and traditional indices of orienting (Bradley, 2009; Bradley et al., 2012; Löw et al., 2008). We hypothesised that if, as the social touch hypothesis suggests, CTs form the first stage of encoding socially relevant and rewarding tactile information, CT 
targeted touch will elicit reflexive autonomic orienting responses, indicated by a decrease in heart rate and increase in SCR in comparison to Control touch. 


\section{Methods}

\subsection{Participants}

Thirty-four participants (mean age 24 years +/- 3.4, 15 female) with no history of psychiatric illness of cardiac abnormalities and normal or corrected to normal vision, took part in exchange for shopping vouchers. Positive And Negative Affect Scale (PANAS; Watson, Clark \& Tellegen, 1985) scores revealed that participants were more positive than negative in their mood state on beginning the study (mean positive mood $=$ $33.56, \mathrm{SD}=5.61$; mean negative $\operatorname{mood}=14.25, \mathrm{SD} 4.39$, scale ranges 10 to 50 ). The study was approved by the LJMU Research Ethics Committee.

\subsection{Materials}

\subsubsection{Face Stimuli}

Twenty-four emotionally neutral female faces (Kramer \& Ward, 2010) were used. The photographs, originally taken against a white background, were cropped with an oval frame that showed just the head and upper shoulders. For all photographs, hair was tied back where necessary, so the full face including the forehead was visible. The resulting images were $800 \times 1132$ pixels in size, and onscreen the faces appeared at a size of approximately $10 \times 14.5 \mathrm{~cm}$.

\subsubsection{Rotary Tactile Stimulator}

Touch was delivered to the volar surface of the left forearm using a rotary tactile stimulator (RTS - Dancer Design). The RTS can deliver touch, using a rotating probe ‘arm', with precise force \& velocity (Essick et al., 1999; Löken et al., 2009). Touch was delivered at a force of $0.3 \mathrm{~N}$ using a probe with a stroking surface measuring 
approximately $10 \times 2 \mathrm{~cm}$, coated in a soft, smooth, synthetic fabric. The RTS interfaced with the PC delivering the conditioning task via parallel port triggers. The two conditions of touch were: CT touch ( $3 \mathrm{~cm} / \mathrm{sec} \times 2$ strokes) and Control touch (30 $\mathrm{cm} / \mathrm{sec} \times 3$ strokes). In both conditions, the RTS stroked backwards and forwards, starting proximally, over an aperture of $8.1 \mathrm{~cm}$. In the CT touch condition, stimulating at $3 \mathrm{~cm} / \mathrm{sec}$ resulted in approximately $5700 \mathrm{~ms}$ of contact time between the RTS probe and the participant's skin. In the Control touch condition at $30 \mathrm{~cm} / \mathrm{sec}$, contact time was approximately $1500 \mathrm{~ms}$.

\subsection{Procedure}

After briefing and consent, participants completed the tasks as follows (see Figure 1): Firstly, seated at a desk, they completed the first iteration of the Ratings Task. After this they were moved to a reclining chair and fitted with the electrodes for the physiological measures, before completing a short questionnaire on their current mood (PANAS; Watson, Clark, \& Tellegen, 1988), after which the experimenter calibrated the RTS. The participants then completed the Conditioning Task. Next, the experimenter removed the electrodes and the participant returned to the desk, where they completed the Dot Probe task, and finally the second iteration of the Ratings Task, before being debriefed.

\subsubsection{Ratings Task}

The ratings task was presented using PsychoPy (Pierce, 2009). First, participants passively viewed all 24 faces, presented in a random order, to reduce the chances that order effects might influence their later judgements. Each face was presented centrally 
for $1000 \mathrm{~ms}$, preceded by a $500 \mathrm{~ms}$ fixation, and followed by a $500 \mathrm{~ms}$ blank screen (see Figure 1, row 1). The participants then viewed an identical presentation of all 24 faces, once more in random order, but this time they were asked after each face, 'How approachable do you find this person?' The participants made their responses by clicking with the mouse on a horizontal, 11 point scale, from 0 'Not at all Approachable', to 10 'Very Approachable'. The second iteration of the ratings task, after Conditioning and Dot Probe tasks, was completed in an identical manner.

\subsubsection{Conditioning Task}

The Conditioning Task and Dot Probe Task were presented using E-Prime 2.0 (Schneider, Eschman, \& Zuccolotto, 2012). For each participant, the eight most neutrally rated faces from the first iteration of the Ratings Task were entered into the Conditioning Task. Four faces were paired with touch at CT optimal speed $(3 \mathrm{~cm} / \mathrm{sec}-$ CT touch) and four with touch at non-CT optimal speed $(30 \mathrm{~cm} / \mathrm{sec}-\mathrm{Control}$ touch). The task consisted of five blocks of sixteen trials each. During a single block, each of the eight faces appeared twice, accompanied by its contingent touch from the RTS. The order of trials was counterbalanced such that the same condition of touch never appeared more than twice in a row. Each trial (see Figure 1, row 2) was initiated by the participant, after which a $2000 \mathrm{~ms}$ fixation appeared, followed by the face which remained on screen for $10,000 \mathrm{~ms}$. The face always appeared slightly to the left or right of centre (order counterbalanced, 50/50 contingency). After $2000 \mathrm{~ms}$ of the face presentation, the RTS would initiate the touch stimulus. The touch stimulus lasted approximately $8000 \mathrm{~ms}$, including inter-stroke intervals. After the face disappeared, there was a $2000 \mathrm{~ms}$ blank screen, followed by the presentation of one of two questions 
(order counterbalanced, 50/50 contingency) - either 'Is that the type of touch you would like to feel again?', or 'Was the face presented to the left or the right?' The participant responded using pre-assigned mouse buttons (left = 'yes' / 'left'; right = 'no' / 'right'). The participant did not know at the start of the trial which question would be asked, thus the task required that they attend to both the face and touch stimulus. There was a minimum inter-trial interval of $9000 \mathrm{~ms}$. Prior to starting the task, participants completed a short practise with non-experimental faces.

\subsubsection{Dot Probe Task}

The Dot Probe Task consisted of four blocks of sixty-four trials. At the start of each trial (see Figure 1, row 3) a fixation cross appeared centrally for $1000 \mathrm{~ms}$, before being replaced by the presentation of two faces, one presented to the left and one to the right of centre, both on the vertical midline. During blocks one and four, the faces remained on screen for $750 \mathrm{~ms}$. During blocks two and three, the faces remained on screen for $1250 \mathrm{~ms}$. The two durations were used to examine whether any effects of attention capture were short, or sustained (eg di Pellegrino, Magarelli, \& Mengarelli, 2011). When the faces cleared, a dot appeared behind either the left or right face, remaining on screen until the participant responded with a key press ( $\mathrm{Z}$ for 'left', $\mathrm{M}$ for 'right'). Participants were asked to respond as quickly and accurately as possible. Importantly, the pair of faces in each trial always consisted of one face that had been paired with CT touch and one face that had been paired with Control touch. The assignment of face condition to side of screen, and the side of screen on which the dot appeared, were counterbalanced and presented quasi-randomly (randomly but accounting for counterbalancing). The task started with four practice trials using non-experimental 
faces.

\subsubsection{Questionnaires}

After completing all the behavioural tasks, the participants completed a set of

psychometric questionnaires. These data are not reported here, but are part of a related study of individual differences in perceptions of touch.

Procedure

\section{Ratings Task 1}

Participants passively view 24 female faces. They then rate each for approachability

\section{Evaluative Conditioning Task}

Eight averagely approachable faces entered into Conditioning Task. Four faces always paired with CT touch ( $3 \mathrm{~cm} / \mathrm{sec}$ ) four with Control touch $(30 \mathrm{~cm} / \mathrm{sec})$

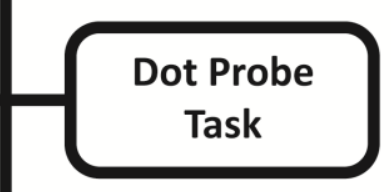

Face pairs in the Dot Probe Task always consist of one face previously paired with CT touch at Conditioning and one previously paired with Control touch

\section{Ratings Task 2}

Participants passively view and then re-rate all 24 faces for approachability

\section{Example Trials}

Ratings Task 1 (one block of 24 trials)

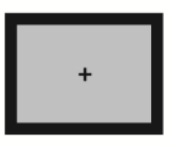

$500 \mathrm{~ms}$
$1000 \mathrm{~ms}$

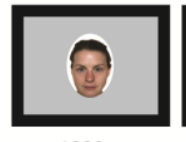

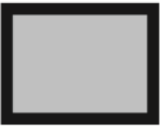

$500 \mathrm{~ms}$

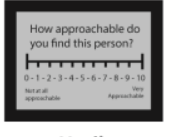

Until

Response
Evaluative Conditioning Task (five blocks of 16 trials)
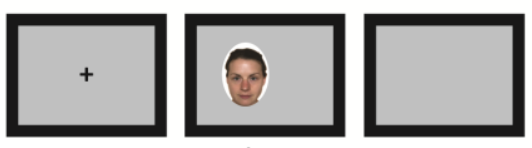

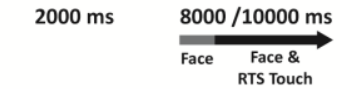

ECG and SCR measures recorded

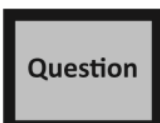

Until Response

$2000 \mathrm{~ms}$

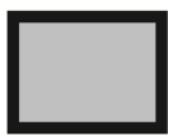

Is that the kind of touch you would like to receive again?' / 'Was the face presented to the left or the right?' click.

Dot Probe Task (four blocks of 64 trials)

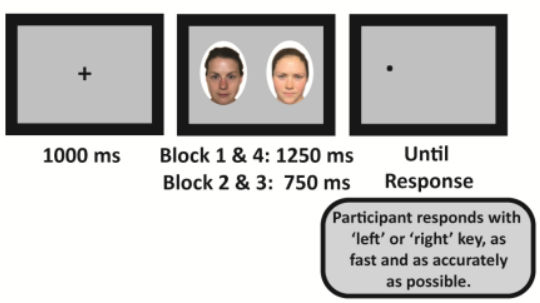

Ratings Task 2 (one block of 24 trials)

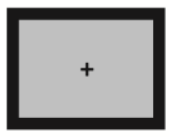

$500 \mathrm{~ms}$

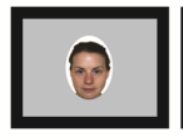

$1000 \mathrm{~ms}$

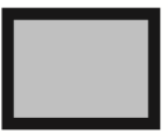

$500 \mathrm{~ms}$

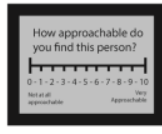

Until 
Figure 1: The procedure each participant undertook during the experiment, including the time course of Trials in the Ratings, Evaluative Conditioning and Dot Probe tasks. Firstly, in the Ratings Task, participants rated the full set of 24 faces for approachability, before completing the Evaluative Conditioning Task, during which they saw their 8 most neutrally rated faces paired with either CT touch or Control touch. After this the participant completed the Dot Probe Task, in which the face pairs consisted of one face previously associated with CT touch and one face previously associated with Control touch. Finally, in a repeat of the Ratings Task, participants rated the full set of faces again for approachability.

\subsection{Psychophysiological Recordings}

Psychophysiological recordings were made using an AD Instruments Power Lab system, with an Octal bio Amp (AD Instruments) used to record the electrocardiogram (ECG), and a specialist amplifier (AD Instruments) used to record skin conductance level. Recordings were sampled at $2000 \mathrm{~Hz}$. The ECG was recorded using a three-lead setup, and filtered online between 0.02 and $100 \mathrm{~Hz}$, with an adaptive mains filter applied. SCR was recorded using a constant current of $22 \mathrm{mV}$, with metal electrodes placed around the fingertips of the left index and middle fingers.

\subsection{Data Treatment and Analysis}

Two participants' data (females, age $=19$ and 25 years) had to be excluded due to failure of the RTS, meaning the final sample size was 32 , which is comparable to previous similar studies (Andreatta \& Pauli, 2015; Petrovic et al., 2008). All data were analysed using SPSS (IBM Corp. Released 2012. IBM SPSS Statistics for Windows, Version 21.0. Armonk, NY: IBM Corp) and R (R Core Team, 2013).

\subsubsection{Face approachability ratings}


Wilks' Lambda multivariate repeated measures ANOVA was used to investigate whether face approachability ratings were altered following conditioning. Initially, the model had two factors, both with two levels: time (ratings obtained pre / post conditioning) and touch (face paired with CT touch / Control touch). Model assumptions were checked by plotting model residuals in combination with verification that the Shapiro-Wilk test of normality for model residuals was not significant. When significant interaction effects were identified, they were further investigated using repeated measures ANOVAs with estimated marginal means comparison, or pairedsamples $t$-tests as appropriate.

Change in face ratings for faces paired with $\mathrm{CT}$ touch and Control touch were compared to the change in ratings produced for the four most neutrally rated faces not used during the conditioning task for each participant (No touch paired faces). Thus, conditioning indices were calculated as the change in approachability ratings for CT touch or Control touch paired faces minus the change in ratings for No touch paired faces:

i) CT touch Conditioning Index: (CT touch After - CT touch Before) - (No touch After - No touch Before)

ii) Control touch Conditioning Index: (Control touch After - Control touch Before) (No touch After - No touch Before)

The resultant $\mathrm{CT}$ touch conditioning index data was found not to be normally distributed due to an outlier (male, age $=29$ years) who's CT touch conditioning index was more than three standard deviations less than the mean. This outlier was removed from the analysis, which allowed the assumption of normality to be met. 


\subsubsection{Dot Probe Task}

Reaction time (RT) data from the Dot Probe Task were cleaned by removing RTs from trials where the participant responded erroneously. Participants made a mean of 2.97 errors $(S D=3.42)$. Following previously published protocols (Bradley, Mogg, \& Lee, 1997; Koster, Crombez, Verschuere, \& De Houwer, 2004), trials where RTs were $<200$ ms or $>2000 \mathrm{~ms}$ were removed (mean $\pm S D$ number of trials per participant removed due to RT being $<200 \mathrm{~ms}: 0.063 \pm 0.25,>2000 \mathrm{~ms}: 0.25 \pm 0.67)$. RT data was found to be positively skewed and thus not normally distributed. To reduce the influence of positive skew, median rather than mean RTs for each participant and condition were calculated, following previously published protocols (Bindemann, Burton, Langton, Schweinberger, \& Doherty, 2007; Moore, Heavey, \& Reidy, 2012). The residuals of the model were found not to be normally distributed and one participant (female, age $=29$ years) was identified as a significant outlier. Examination of model residuals identified this participant's residuals for all 8 conditions were more than three standard deviations from the mean and for half of these conditions, the residuals for this participant were more than 5 standard deviations from the mean. This participant was therefore removed from the analysis (N.B. this was not the same participant as the outlier identified in section 2.5.1.). Additionally, data were square root transformed. Model residuals then met the assumption of normality. Data were analysed using Wilks' Lambda multivariate repeated measures ANOVAs.

\subsubsection{ECG}

ECG and SCR data were missing for one participant due to a corrupted file (male, age = 20 years), so datasets from 31 participants were analysed for both measures. The ECG 
data were filtered using a 0.1 - $30 \mathrm{~Hz}$ bandpass filter and an $\mathrm{R}$ peak finder was used to generate inter-beat intervals (IBIs). Change scores in IBI were created on a trial by trial basis, using the final two beats before the onset of the face during the conditioning task as a baseline (adapted from Gunther Moor, Crone, \& Van Der Molen, 2010). This resulted in positive changes denoting a slowing of heart rate and negative changes a speeding up of heart rate. Artefacts were then removed on a participant by participant basis by generating boundaries of three standard deviations above and below the mean change score for every participant, including all beats across all trials in this calculation. Any trial that contained an IBI change that violated these boundaries was then removed from the analysis. This resulted in the removal of an average of $1 \%$ of trials ( $\mathrm{Min}=0 \%$, Max $=5 \%$ ). The IBI data were analysed in terms of mean amplitudes, using repeated measures ANOVAs. To divide the IBI data from each trial in terms of time, beat numbers 1 and 2 after the fixation period were analysed as responses to the face only, and beats $3-8$ were analysed as responses to the touch stimulus.

\subsubsection{SCR}

Two separate examinations of the SCR data were made. The first examined skin responses to the presentation of the face, before the touch stimulus, in the conditioning task. The maximum value of the skin conductance level during the $1000 \mathrm{~ms}$ prior to the onset of the face on each trial was sampled - i.e the maximum value of the final 1000 $\mathrm{ms}$ of the fixation period. Next, the maximum value of the period from $1000 \mathrm{~ms}$ to $5000 \mathrm{~ms}$ (in accordance with sampling guidelines from Boucsein et al., 2012; Dawson, Schell, \& Filion, 2007, p. 225) after the onset of the face was sampled. A change score was calculated between these values by subtracting the baseline maximum from the 
maximum of the window of interest. Only trials where this value exceeded 0.01 microsiemens were considered to contain responses and were included in analyses. An average of $68 \%$ of trials contained responses during this period $(\operatorname{Min}=33, \operatorname{Max}=88)$. Two participants' data (male, age $=19$ years; female, age $=25$ years) had to be removed from this analysis, because they did not generate enough responses to complete one cell of the ANOVA. The second examination was of the period after the touch stimulation had begun. The same procedure was applied, this time with the baseline maximum taken from the final $1000 \mathrm{~ms}$ of the period where the face was onscreen pre-touch, and change scores calculated between this value and the maximum value from the period 1000 to $5000 \mathrm{~ms}$ after touch onset. During this period $80 \%$ of trials contained responses of over 0.01 microsiemens. The data from both examinations were analysed in separate repeated measures ANOVAs, with Speed of touch and Block as factors. 


\section{Results}

3.1 Comparison of the effect of conditioning with CT touch vs Control touch on face approachability ratings.

Analysis of change in face approachability ratings following conditioning with CT touch compared to Control touch identified a significant Touch $\mathrm{x}$ Task interaction $\left(F_{1,31}=8.316\right.$, $p=0.007$, partial $\eta^{2}=0.212$ ), as presented in Figure 2A. Before conditioning, face ratings were comparable (CT touch paired faces: $M=5.52, S D=0.83$; Control touch paired faces: $M=5.51, S D=0.87), t_{31}=0.226, p=0.823$. After conditioning, faces paired with Control touch $(M=5.13, S D=1.28)$ were rated significantly less approachable than those paired with CT touch $(M=5.55, S D=1.23), t_{31}=3.064, p=0.004$.

To further investigate this interaction effect, we next compared changes in face ratings obtained following conditioning, to ratings changes for four faces that were initially equally neutrally rated but not included in the conditioning task. A paired samples $t$-test revealed average ratings for the touch paired faces before conditioning $(M=5.51, S D=$ $0.85)$ were not significantly different to the No touch paired faces used in this analysis $\left.(M=5.54, S D=0.80), \mathrm{t}_{31}=-0.523, p=0.605\right)$. A significant Time $\mathrm{x}$ Touch interaction was identified $\left(F_{2,30}=4.842, p=0.015\right.$, partial $\left.\eta^{2}=0.244\right)$, as presented in Figure 2A. 


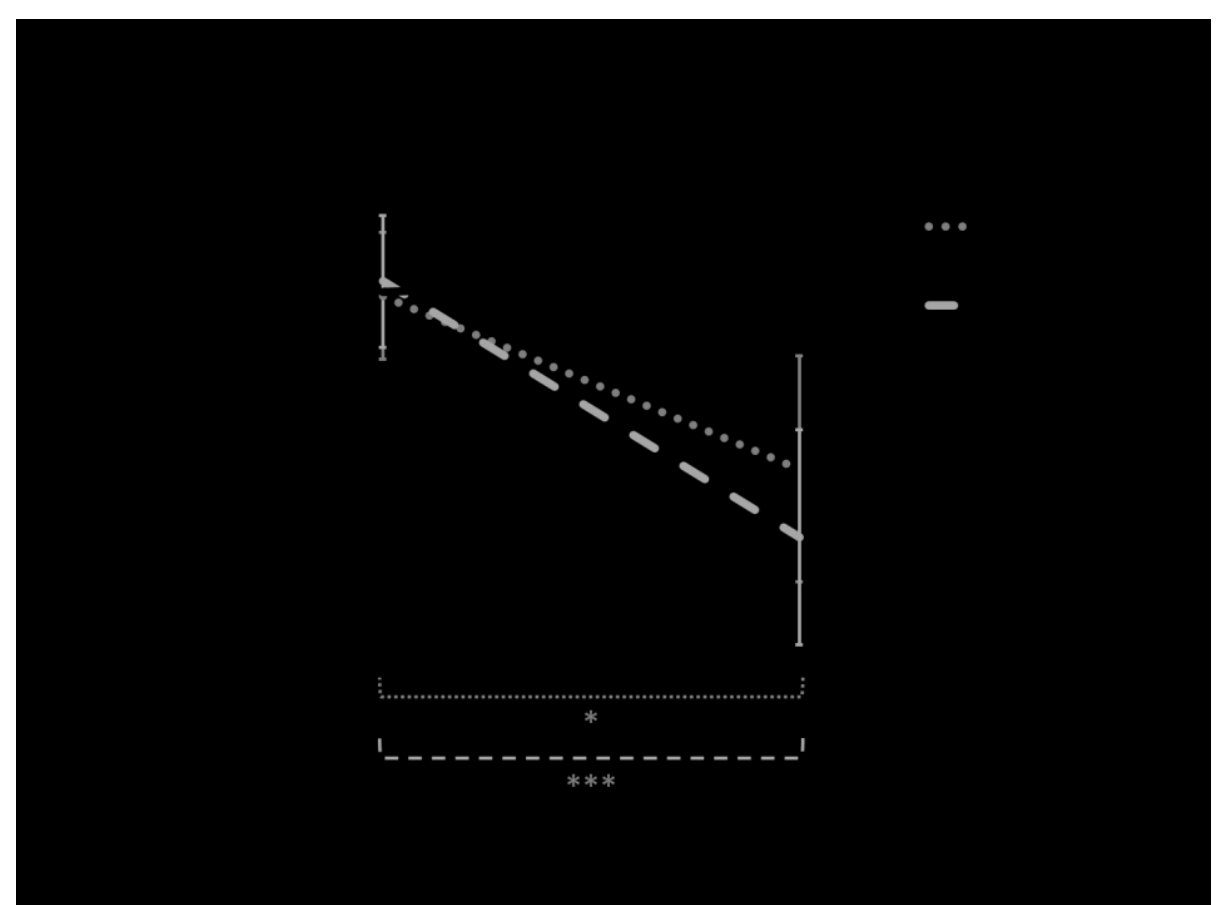

Figure 2A: Change in face approachability ratings following conditioning. Before conditioning, all face ratings were comparable. After conditioning faces paired with CT Touch were rated significantly more approachable than both Control touch and No-touch paired faces. For CT touch paired faces, ratings were comparable before and after conditioning, whereas for Control touch \& No touch paired faces, approachability ratings decreased following conditioning. Mean $\pm 95 \%$ confidence intervals are shown. $* p<0.05, * * p<0.01$, *** $p<0.001$.

Before conditioning, no differences were identified in approachability ratings for the faces in the three touch conditions $\left(F_{2,30}=0.146, p=0.865\right.$, partial $\left.\eta^{2}=0.010\right)$. After conditioning, a significant difference was identified $\left(F_{2,30}=5.603, p=0.009\right.$, partial $\eta^{2}=$ 0.272). CT touch paired faces were rated significantly more approachable than either the Control touch $\left(t_{31}=3.058, p=0.004\right)$ or the No touch paired faces $(M=4.98, S D=0.79$, $\left.\mathrm{t}_{31}=2.639, p=0.013\right)$. Faces paired with Control touch and No touch paired faces were comparably approachable $\left(t_{31}=0.736, p=0.467\right)$.

To compare the conditioning effect induced by CT touch to that produced by Control touch, conditioning indices were calculated (see section 2.4.1). The results 
obtained are presented in Figure 2B. A paired samples $t$-test identified the CT touch conditioning index $(M=0.73, S D=1.02)$ to be significantly greater than the Control touch conditioning index $(M=0.27, S D=0.91), t_{30}=3.155, p=0.004$. This effect was also significant before removing the outlier identified in section $2.5 .1\left(t_{31}=2.884, p=\right.$ $0.007)$.

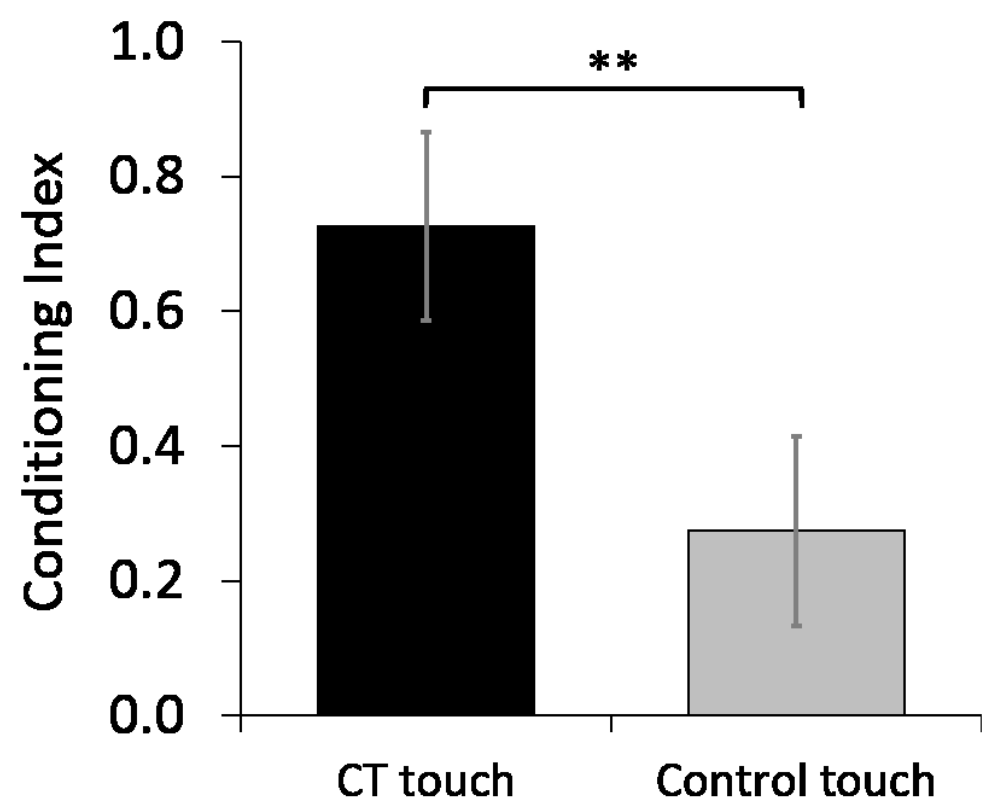

Figure 2B: CT touch induces a significantly greater conditioning effect on face approachability ratings than Control touch. Change in approachability rating of faces not used in the conditioning task, but rated before and after, was subtracted from the change in face rating induced by CT touch (CT conditioning index) and Control touch (Control conditioning index). The CT conditioning index was significantly greater than the Control conditioning index. Mean $\pm 95 \%$ confidence intervals are shown. ${ }^{* *} p=$ 0.004 .

\subsection{Dot Probe}

A within-subjects ANOVA was used to investigate the median RTs obtained from the dot probe task. The analysis design had 3 factors, each with two levels: Touch (CT / Control), Latency (slow, fast) and Position (left, right). Touch referred to whether the dot probe appeared in the position of the $\mathrm{CT}$ touch paired face or the Control touch paired face. 
Latency referred to how long the faces were presented before being replaced by the dot probe (stimulus onset asynchrony (SOA)). For the fast condition, SOA was $750 \mathrm{~ms}$. For the slow condition, SOA was $1250 \mathrm{~ms}$. Position refers to whether the dot probe appeared on the left or right of the screen. No significant effects were identified, although the effect of touch approached significance $\left(F_{1,30}=3.627, p=0.066\right.$, partial $\left.\eta^{2}=0.108\right)$, with square root reaction time being greater for $\mathrm{CT}$ touch paired faces $(M=19.30, S D=0.99)$ than Control touch paired faces $(M=19.23, S D=0.95)$ and this data is presented in Figure 3. Before removal of the outlier identified in section 2.5.2, this effect of touch was also nonsignificant $\left(F_{1,31}=2.363, p=0.134\right.$, partial $\left.\eta^{2}=0.071\right)$.

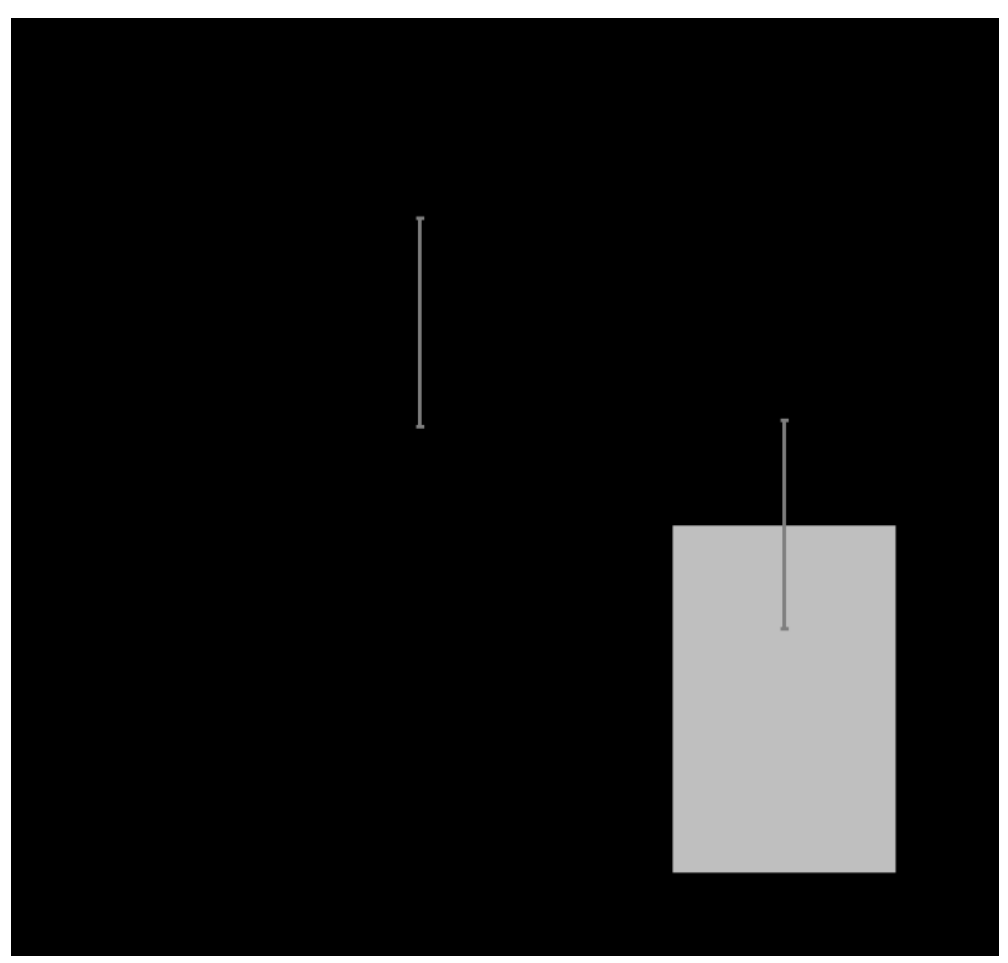

Figure 3: Square root reaction time to the dot probe when it appeared in the position of the CT touch paired face (CT touch) compared to when it appeared in the position of the Control touch paired face (Control touch). Mean $\pm 95 \%$ confidence intervals are shown.

\subsection{ECG data}


Initially the ECG data were entered into a repeated measures ANOVA, with the withinsubjects factors of Touch (CT touch, Control touch), Time (face period (beats 1 and 2), face and touch period (beats 3 to 8$)$ ), and $\operatorname{Block}(1,2,3,4,5)$. There was a significant main effect of Touch, $\left(F_{1,30}=4.2, p=0.048, \eta^{2}=.12\right)$, with $\mathrm{CT}$ touch producing a significantly greater deceleration of the heart than Control touch. There was also a significant Touch $\mathrm{x}$ Time interaction, $\left(F_{1,30}=6.6, p=0.016, \eta^{2}=.18\right)$. This reflects the fact that during the first period of the trial, where only the face was present, there was no significant difference in deceleration for the two touch conditions, $t_{30}=0.38, p=0.71$. However, for the period of trial where touch was being applied, heart rate deceleration was significantly greater for CT than Control touch $\left(t_{30}=3.0, p=0.005\right)$, see Figure 4 .

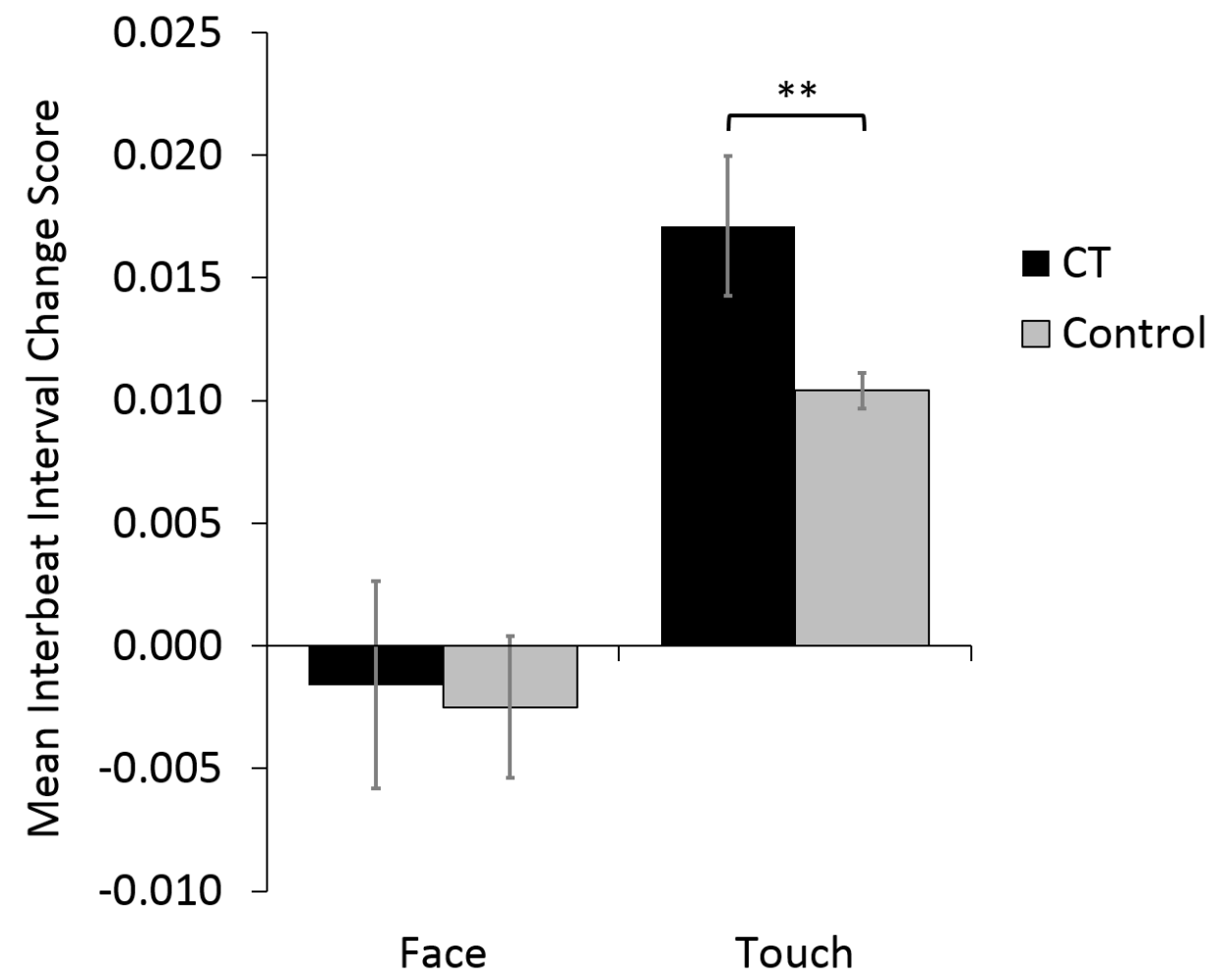

Figure 4: Mean change in interbeat interval (IBI) during trials of the Evaluative Conditioning Task. The bars in the left hand column represent the mean change from baseline in the first two IBIs of the trial, taken to represent change caused by the face stimulus alone. The bars in the right hand column represent the mean change in IBI during beats three to eight, during the application of touch. Mean $\pm 95 \%$ confidence intervals are shown. ${ }^{* *} p<.01$ for difference between CT touch and Control touch conditions. 


\subsection{SCR Results}

The SCR data from the period where the participant observed the face alone prior to touch, were entered into a repeated measures ANOVA, with the within-subjects factors of Touch (CT touch \& Control touch) and Block $(1,2,3,4,5)$. The analysis of this period revealed no significant main effects or interactions.

The SCR data from the period where the participant observed the face and received the touch stimulus (see Figure 5) were entered into a repeated measures ANOVA, with the within-subjects factors of Touch (CT touch \& Control touch) and Block $(1,2,3,4,5)$. The analysis revealed only a main effect of Touch $\left(F_{1,30}=6.5, p=0.016, \eta^{2}=.18\right)$, with significantly larger SCRs in response to Control than CT touch.

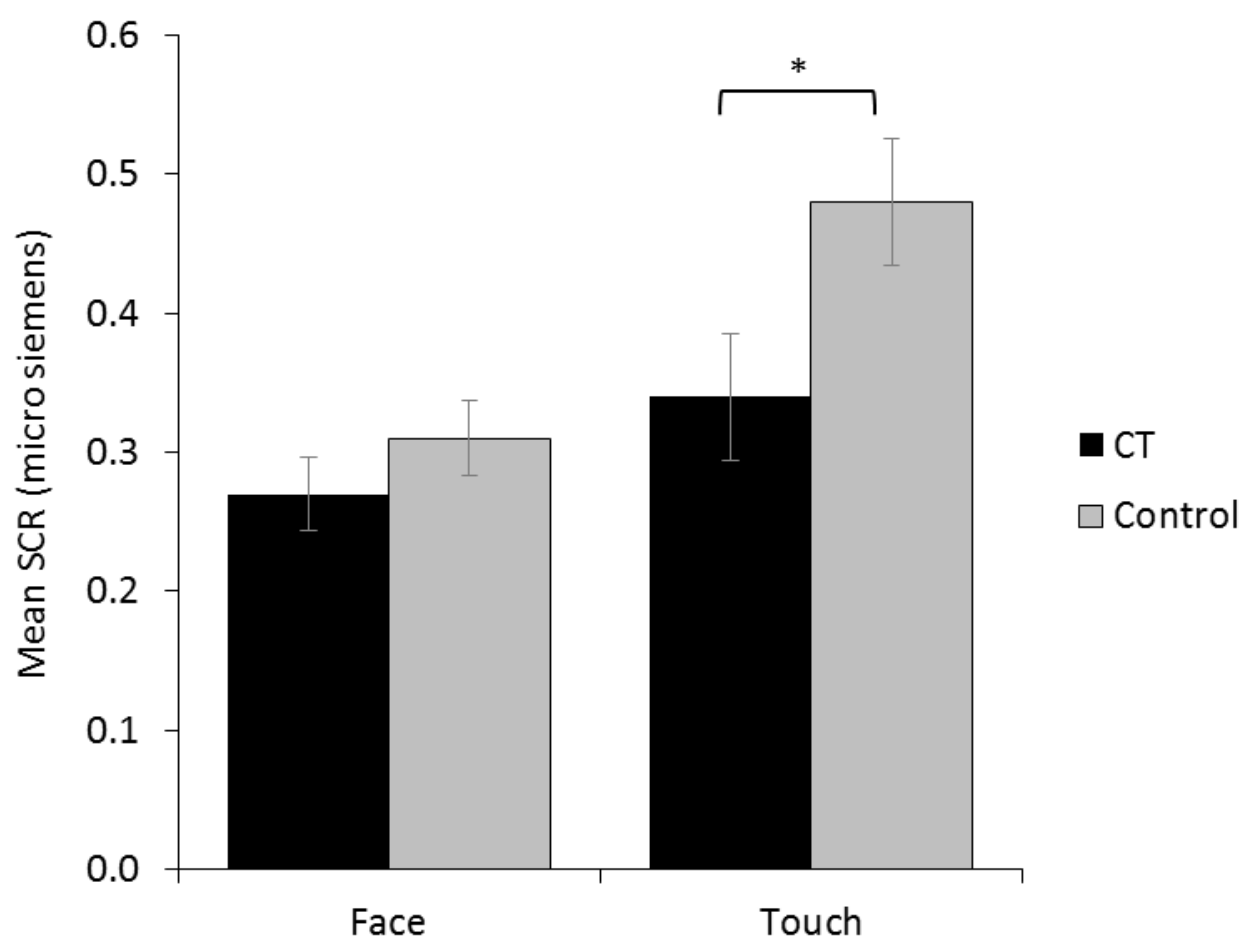

Figure 5: Mean change in Skin Conductance Response (SCR), during the Evaluative Conditioning Task, to the initial appearance of the face (bars in left hand column), and 
the application of touch (bars in right hand column). Mean $\pm 95 \%$ confidence intervals are shown. ${ }^{*} p<.05$ for the difference between CT touch and Control touch conditions. 


\section{Discussion}

To date, the majority of conditioning studies conducted in humans have used aversive rather than appetitive stimuli (for review see Hofmann et al., 2010), though see (Andreatta \& Pauli, 2015). While previous studies have reported conditioning effects to a painful cutaneous stimulus (Petrovic et al., 2008), in the present study we used a tactile stimulus as an appetitive unconditioned stimulus. Specifically, in support of the social touch hypothesis (Morrison et al., 2010; Olausson et al., 2010), we have shown that touch, designed to optimally activate CTs, carries a positive affective value that can be passed to previously neutral stimuli through repeated co-occurrence. While all faces used in the study were rated equally approachable prior to conditioning, post conditioning faces paired with CT touch were significantly more approachable than faces paired with Control touch or those not paired with any type of touch.

It is noteworthy that in the present study, we did not see a significant increase in ratings of approachability of $\mathrm{CT}$ touch paired faces, rather these faces did not show the same reduction in approachability as the other faces showed over the course of the study. This finding is consistent with those of Triscoli, Ackerley, \& Sailer (2014) who reported that after 50 minutes of repeated stimulation, though pleasantness ratings of both types of touch declined, ratings of stroking at CT optimal velocity remained significantly higher than for faster, Non-CT optimal touch. However, the fact that in the present study there was also a drop-in approachability of No touch paired faces suggests that it was not the repetitive touch per se, but perhaps a reduction in the affective state of the participants over the course of the experimental session that reduced their approachability ratings of all but the CT touch paired faces. Further work is needed to determine explicitly what 
impact the affective state of a participant has on both the conscious appraisal and implicit impact of touch. For example, in a context of social isolation or stress, we would predict CT activating touch would be perceived as more rewarding, where it can be seen functionally as helping to correct a need state (Cabanac, 1971; Ellingsen, Leknes, Løseth, Wessberg, \& Olausson, 2016; Morrison, 2016).

Consistent with previous studies, we saw a significantly greater deceleration in heart rate in response to CT versus Control touch (Fairhurst et al., 2014; Pawling et al., 2017). Greater cardiac deceleration has previously been reported in response to emotional versus neutral stimuli and is a classic component of the orienting response to motivationally salient stimuli (Bradley et al., 2001; Bradley et al., 2012; Lang \& Bradley, 2010). However, in contrast to our hypothesis, while both types of touch elicited electrodermal responses, the SCR response to Control touch was significantly higher than to CT touch. Taken together, this suggests that while CT touch is more positively valenced, the Control touch was more arousing (Bradley et al., 2001). There was no significant effect of time in our measures of physiological responses, thus faces did not come to elicit the physiological responses elicited by the touch with which they were associated. This is not unexpected as evaluative conditioning is qualitatively distinct from classical conditioning and expectancy learning does not take place (Baeyens \& De Houwer, 1995). Thus, unlike Pavlovian fear conditioning for example, a change in response to the conditioned stimuli can only generally be seen by using direct measures of preference or evaluation, such as approachability ratings (Baeyens \& De Houwer, 1995). 
The dot probe task was included in the present study as an implicit measure of the relative attentional salience of the touch paired faces (Frewen, Dozois, Joanisse, \& Neufeld, 2008; Koster et al., 2004; Moore et al., 2012). However, we found no significant effect of touch on task performance. In fact, in contrast to our hypothesis, on average, reaction times were faster on trials when the probe appeared behind Control than CT touch paired faces. This observation is consistent with the significantly higher arousal elicited by the Control touch (Pool et al., 2016). The fact we saw conditioning effects with our explicit but not implicit behavioural measure is in-line with previous literature, where evaluative conditioning effects are weaker and less consistent for implicit than explicit measures (see Hofmann et al., 2010 for meta-analysis).

Our finding that somatosensory signals can influence affective processing is consistent with a previous study by Schirmer et al (2011), which reported that a brief, socially relevant tactile stimulus (light pressure applied to the forearm) modulated neural responses to affective visual images, irrespective of whether it was delivered by, or attributable to, a close friend or a computer controlled device. However, in order to elicit such effects, touch may need to have some social relevance, as in another recent study a vibro-tactile stimulus applied to the palm of the hand was not found to modulate neural or physiological responses to a range of emotional faces (Spapé, Harjunen, \& Ravaja, 2017).

While the present study was designed with a bottom-up approach, predicated on the differential response characteristics of myelinated and unmyelinated mechanosensory afferents, we acknowledge that in the real world, top-down effects, such as the context 
in which social touch is experienced, will have a significant impact on how it is evaluated (Ellingsen et al., 2016; Gazzola et al., 2012; Suvilehto, Glerean, Dunbar, Hari, \& Nummenmaa, 2015). For example, Gazzola et al (2012) reported that the same tactile stimulus elicited distinct neural responses depending on whether heterosexual male participants believed they were being touched by a male or female experimenter. Furthermore, Croy, Drechsler, Hamilton, Hummel, \& Olausson, (2016) found neural responses to $\mathrm{CT}$ activating touch were modulated by the affective value of the broader sensory context it was delivered within. In their study, concurrent presentation of touch with an unpleasant odour dampened responses in affect related brain regions, including posterior insula cortex. Behaviourally, in the visual domain, Ellingsen et al (2014) found that touch was rated as significantly more pleasant when presented with a smiling versus a frowning face. Further work is needed to explicitly test the impact of social context on behavioural and physiological responses to CT targeted touch (Ellingsen et al., 2016).

It should be noted that the stimuli used in the present study not only differed in terms of stroking velocity but also in terms of contact time on the skin, with the CT targeted touch trials involving four times as much skin contact as Control touch. This is consistent with previous research in this area, where the focus is on the bottom-up effects of tactile stimuli (Ackerley et al., 2014; Björnsdotter, Löken, Olausson, Vallbo, \& Wessberg, 2009; Croy, Geide, Paulus, Weidner, \& Olausson, 2016; McGlone et al., 2012; Morrison, Bjornsdotter, \& Olausson, 2011; Perini et al., 2015). To this end, stimuli are delivered at velocities designed to differentially activate cutaneous afferent nerves, and tend to be matched for the number of times the stimulus moves across the 
receptive field of the nerves, rather than time in contact with the skin (though see Crucianelli, Metcalf, Fotopoulou, \& Jenkinson, 2013; Kaiser et al., 2015; Lloyd, Gillis, Lewis, Farrell, \& Morrison, 2013; Voos, Pelphrey, \& Kaiser, 2013 for time matched CT targeted stimulation). In a recent study, we included two control conditions, one where stimuli were matched in terms of strokes and another where they were matched for skin contact time (Pawling et al., 2017). Across both behavioural and physiological measures used, we saw no significant differences between these two control conditions, with both producing significantly lower hedonic ratings and smaller decreases in heart rate than the CT targeted touch. Thus, while it is possible that the longer contact could account for the differential behavioural and physiological effects reported here, based on previous findings, we believe it is unlikely.

A further limitation of the present study is the fact we did not explicitly ask participants if they were aware of the contingencies in the conditioning task. While contingency awareness is an important moderator of evaluative conditioning effects, explicit knowledge of the relationship between the CS and US is not necessary for conditioning to take place (Hofmann et al., 2010). With 4 different CSs for each of our two USs, the relationship is likely to have been harder for participants to learn explicitly than other studies with half the number of conditioned stimuli (eg Andreatta \& Pauli, 2015; Petrovic et al., 2008). In addition, since both our tactile stimuli are rated as moderately pleasant, participants would have been unlikely to guess the study hypothesis and make their responses accordingly. Finally, given evaluations of the eight experimental faces were always made in the context of rating sixteen other task irrelevant faces, conscious 
recall of which faces were paired with which specific type of stroking touch seems highly improbable.

In conclusion, the findings from the present study provide further support for the social touch hypothesis (Morrison et al., 2010; Olausson et al., 2010), by showing that touch, specially targeted to optimally activate CTs, has a positive affective value that can be acquired by previously neutral stimuli it is associated with. Additionally, the pattern of responses observed from our combined measures of sympathetic and parasympathetic nervous system activity add further support to the hypothesis that touch targeted to optimally activate CTs is of specific social relevance and thus has a salience which captures attention (Bradley et al., 2001; Bradley et al., 2012; Lang \& Bradley, 2010; Pawling et al 2017). In evolutionary terms, CT activating touch is likely to have gained its rewarding value because it signals the proximity of others, thereby conferring a survival benefit (Morrison, 2016). 


\section{Acknowledgements}

This work was supported by The Leverhulme Trust, grant number RPG-2013-058

awarded to FM \& SW. The funders had no role in study design, data collection and analysis, decision to publish, or preparation of the manuscript. 


\section{References}

Ackerley, R., Backlund Wasling, H., Liljencrantz, J., Olausson, H., Johnson, R. D., \& Wessberg, J. (2014). Human C-Tactile Afferents Are Tuned to the Temperature of a Skin-Stroking Caress. Journal of Neuroscience, 34(8), 2879-2883.

http://doi.org/10.1523/JNEUROSCI.2847-13.2014

Andreatta, M., \& Pauli, P. (2015). Appetitive vs. Aversive conditioning in humans.

Frontiers in Behavioral Neuroscience, 9(MAY), 128.

http://doi.org/10.3389/fnbeh.2015.00128

Baeyens, F., \& De Houwer, J. (1995). Evaluative conditioning is a qualitatively distinct form of classical conditioning: a reply to Davey (1994). Behaviour Research and

Therapy, 33(7), 825-831. http://doi.org/10.1016/0005-7967(95)00021-O

Bindemann, M., Burton, A. M., Langton, S. R. H., Schweinberger, S. R., \& Doherty, M.

J. (2007). The control of attention to faces. Journal of Vision, 7(10), 15.

Björnsdotter, M., Löken, L., Olausson, H., Vallbo, A., \& Wessberg, J. (2009).

Somatotopic organization of gentle touch processing in the posterior insular cortex.

The Journal of Neuroscience : The Official Journal of the Society for

Neuroscience, 29(29), 9314-9320. http://doi.org/10.1523/JNEUROSCI.0400-

09.2009

Boucsein, W., Fowles, D. C., Grimnes, S., Ben-Shakhar, G., Roth, W. T., Dawson, M.

E., \& Filion, D. L. (2012). Publication recommendations for electrodermal measurements. Psychophysiology, 49, 1017-1034. http://doi.org/10.111/j.14698986.2012.01384.x

Bradley, B. P., Mogg, K., \& Lee, S. C. (1997). University of Cambridge, Cambridge, U.K. (Received 14 April 1997), 35(10). 
Bradley, M. M. (2009). Natural selective attention: Orienting and emotion. Psychophysiology, 46(1), 1-11. http://doi.org/10.1111/j.1469-8986.2008.00702.x

Bradley, M. M., Codispoti, M., Sabatinelli, D., \& Lang, P. J. (2001). Emotion and motivation I: Defensive and Appetitive Reactions in Picture Processing. Emotion (Washington, D.C.), 1(3), 276-298. http://doi.org/10.1037/1528-3542.1.3.276

Bradley, M. M., Keil, A., \& Lang, P. J. (2012). Orienting and emotional perception: Facilitation, attenuation, and interference. Frontiers in Psychology, 3(NOV), 1-6. http://doi.org/10.3389/fpsyg.2012.00493

Cabanac, M. (1971). Physiological Role of Pleasure A stimulus can feel pleasant or unpleasant depending upon its usefulness as determined by internal signals . Science, 173(4002), 1103-1107. http://doi.org/10.1126/science.173.4002.1103

Cacioppo, J. T., \& Tassinary, L. G. (1992). Unobservable facial actions and emotion. Psychological Science, 3, 28-33.

Croy, I., Drechsler, E., Hamilton, P., Hummel, T., \& Olausson, H. (2016). Olfactory modulation of affective touch processing - A neurophysiological investigation. NeuroImage, 135, 135-141. http://doi.org/10.1016/j.neuroimage.2016.04.046

Croy, I., Geide, H., Paulus, M., Weidner, K., \& Olausson, H. (2016). Affective touch awareness in mental health and disease relates to autistic traits - An explorative neurophysiological investigation. Psychiatry Research, 245, 491-496. http://doi.org/10.1016/j.psychres.2016.09.011

Crucianelli, L., Metcalf, N. K., Fotopoulou, A. (Katerina), \& Jenkinson, P. M. (2013). Bodily pleasure matters: Velocity of touch modulates body ownership during the rubber hand illusion. Frontiers in Psychology, 4(October), 1-7. http://doi.org/10.3389/fpsyg.2013.00703 
Dawson, M. E., Schell, A. M., \& Filion, D. L. (2007). The Electrodermal System. The Handbook of Psychophysiology. http://doi.org/10.1016/j.ces.2007.04.037

De Houwer, J., Thomas, S., \& Baeyens, F. (2001). Associative learning of likes and dislikes: a review of 25 years of research on human evaluative conditioning. Psychological Bulletin, 127(6), 853-869. http://doi.org/10.1037//D0332909.127.6.853

di Pellegrino, G., Magarelli, S., \& Mengarelli, F. (2011). Food pleasantness affects visual selective attention. Quarterly Journal of Experimental Psychology, 64(3), 560-571. http://doi.org/10.1080/17470218.2010.504031

Ditzen, B., Neumann, I. D., Bodenmann, G., von Dawans, B., Turner, R. a., Ehlert, U., \& Heinrichs, M. (2007). Effects of different kinds of couple interaction on cortisol and heart rate responses to stress in women. Psychoneuroendocrinology, 32(5), 565-574. http://doi.org/10.1016/j.psyneuen.2007.03.011

Dunbar, R. I. M. (2010). The social role of touch in humans and primates: Behavioural function and neurobiological mechanisms. Neuroscience and Biobehavioral Reviews, 34(2), 260-268. http://doi.org/10.1016/j.neubiorev.2008.07.001

Ellingsen, D. M., Leknes, S., Løseth, G., Wessberg, J., \& Olausson, H. (2016). The neurobiology shaping affective touch: Expectation, motivation, and meaning in the multisensory context. Frontiers in Psychology, 6(JAN), 1-16. http://doi.org/10.3389/fpsyg.2015.01986

Ellingsen, D. M., Wessberg, J., Chelnokova, O., Olausson, H., Laeng, B., \& Leknes, S. (2014). In touch with your emotions: Oxytocin and touch change social impressions while others' facial expressions can alter touch. Psychoneuroendocrinology, 39(1), 11-20. 
http://doi.org/10.1016/j.psyneuen.2013.09.017

Essick, G. K., James, A., \& McGlone, F. P. (1999). Psychophysical assessment of the affective components of non-painful touch. Neuroreport, 10(10), 2083-2087. http://doi.org/10.1097/00001756-199907130-00017

Essick, G. K., McGlone, F., Dancer, C., Fabricant, D., Ragin, Y., Phillips, N., ... Guest, S. (2010). Quantitative assessment of pleasant touch. Neuroscience and Biobehavioral Reviews, 34(2), 192-203. http://doi.org/10.1016/j.neubiorev.2009.02.003

Fairhurst, M. T., Löken, L., \& Grossmann, T. (2014). Physiological and behavioral responses reveal 9-month-old infants' sensitivity to pleasant touch. Psychological Science, 25(5), 1124-1131. http://doi.org/10.1177/0956797614527114

Frewen, P. A., Dozois, D. J. A., Joanisse, M. F., \& Neufeld, R. W. J. (2008). Selective attention to threat versus reward: meta-analysis and neural-network modeling of the dot-probe task. Clinical Psychology Review, 28(2), 307-337. http://doi.org/10.1016/j.cpr.2007.05.006

Gazzola, V., Spezio, M. L., Etzel, J. a., Castelli, F., Adolphs, R., \& Keysers, C. (2012). Primary somatosensory cortex discriminates affective significance in social touch. Proceedings of the National Academy of Sciences, 109(25), E1657-E1666. http://doi.org/10.1073/pnas.1113211109

Gordon, I., Voos, A. C., Bennett, R. H., Bolling, D. Z., Pelphrey, K. a., \& Kaiser, M. D. (2013). Brain mechanisms for processing affective touch. Human Brain Mapping, 34(4), 914-922. http://doi.org/10.1002/hbm.21480

Grewen, K. M., Girdler, S. S., Amico, J., \& Light, K. C. (2005). Effects of partner support on resting oxytocin, cortisol, norepinephrine, and blood pressure before 
and after warm partner contact. Psychosomatic Medicine, 67(4), 531-538. http://doi.org/10.1097/01.psy.0000170341.88395.47

Gunther Moor, B., Crone, E. A., \& Van Der Molen, M. W. (2010). The Heartbrake of Social Rejection. Psychological Science, 21(9), 1326-1333. http://doi.org/10.1177/0956797610379236

Hofmann, W., De Houwer, J., Perugini, M., Baeyens, F., \& Crombez, G. (2010). Evaluative conditioning in humans: A meta-analysis. Psychological Bulletin, 136(3), 390-421. http://doi.org/10.1037/a0018916

Kaiser, M. D., Yang, D. Y.-J., Voos, A. C., Bennett, R. H., Gordon, I., Pretzsch, C., ... Pelphrey, K. a. (2015). Brain Mechanisms for Processing Affective (and Nonaffective) Touch Are Atypical in Autism. Cerebral Cortex (New York, N.Y. : 1991), (2002), bhv125-. http://doi.org/10.1093/cercor/bhv125

Koster, E. H. W., Crombez, G., Verschuere, B., \& De Houwer, J. (2004). Selective attention to threat in the dot probe paradigm: Differentiating vigilance and difficulty to disengage. Behaviour Research and Therapy, 42, 1183-1192. http://doi.org/10.1016/j.brat.2003.08.001

Kramer, R. S., \& Ward, R. (2010). Internal facial features are signals of personality and health. Quarterly Journal of Experimental Psychology, 63(11), 2273-2287. http://doi.org/10.1080/17470211003770912

Lang, P. J., \& Bradley, M. M. (2010). Emotion and the motivational brain. Biological Psychology, 84(3), 437-450. http://doi.org/10.1016/j.biopsycho.2009.10.007

Larsen, J. T., Norris, C. J., \& Cacioppo, J. T. (2003). Effects of positive and negative affect on electromyographic activity over zygomaticus major and .... Psychophysiology, 40(5), 776-785. http://doi.org/10.1111/1469-8986.00078 
Light, K. C., Grewen, K. M., \& Amico, J. a. (2005). More frequent partner hugs and higher oxytocin levels are linked to lower blood pressure and heart rate in premenopausal women. Biological Psychology, 69(1 SPEC. ISS.), 5-21. http://doi.org/10.1016/j.biopsycho.2004.11.002

Lloyd, D. M., Gillis, V., Lewis, E., Farrell, M. J., \& Morrison, I. (2013). Pleasant touch moderates the subjective but not objective aspects of body perception. Frontiers in Behavioral Neuroscience, 7(December), 207. http://doi.org/10.3389/fnbeh.2013.00207

Löken, L. S., Wessberg, J., Morrison, I., McGlone, F., \& Olausson, H. (2009). Coding of pleasant touch by unmyelinated afferents in humans. Nature Neuroscience, 12(5), 547-548. http://doi.org/10.1038/nn.2312

Loseth, G. E., Ellingsen, D.-M., \& Leknes, S. (2014). State-dependent $\mu$-opioid modulation of social motivation. Frontiers in Behavioral Neuroscience, 8(December), 430. http://doi.org/10.3389/fnbeh.2014.00430

Löw, A., Lang, P. J., Smith, J. C., Bradley, M. M., Low, A., Lang, P. J., ... Bradley, M. M. (2008). Emotional Arousal in Threat and Reward. Psychological Science, 19(9), 865-873.

Maruyama, K., Shimoju, R., Ohkubo, M., Maruyama, H., \& Kurosawa, M. (2012). Tactile skin stimulation increases dopamine release in the nucleus accumbens in rats. Journal of Physiological Sciences, 62(3), 259-266.

http://doi.org/10.1007/s12576-012-0205-z

Mcglone, F., Olausson, H., Boyle, J. a., Jones-Gotman, M., Dancer, C., Guest, S., \& Essick, G. (2012). Touching and feeling: Differences in pleasant touch processing between glabrous and hairy skin in humans. European Journal of Neuroscience, 
35(11), 1782-1788. http://doi.org/10.1111/j.1460-9568.2012.08092.x

McGlone, F., Wessberg, J., \& Olausson, H. (2014). Discriminative and Affective Touch: Sensing and Feeling. Neuron, 82(4), 737-755.

http://doi.org/10.1016/j.neuron.2014.05.001

Moore, D. J., Heavey, L., \& Reidy, J. (2012). Attentional processing of faces in ASD: a dot-probe study. Journal of Autism and Developmental Disorders, 42(10), 2038 2045.

Morrison, I. (2016). Keep Calm and Cuddle on: Social Touch as a Stress Buffer. Adaptive Human Behavior and Physiology, 344-362. http://doi.org/10.1007/s40750-016-0052-x

Morrison, I., Bjornsdotter, M., \& Olausson, H. (2011). Vicarious Responses to Social Touch in Posterior Insular Cortex Are Tuned to Pleasant Caressing Speeds. Journal of Neuroscience, 31(26), 9554-9562. http://doi.org/10.1523/JNEUROSCI.0397-11.2011

Morrison, I., Löken, L. S., \& Olausson, H. (2010). The skin as a social organ. Experimental Brain Research, 204(3), 305-314. http://doi.org/10.1007/s00221009-2007-y

Olausson, H., Cole, J., Rylander, K., McGlone, F., Lamarre, Y., Wallin, B. G., ... Vallbo, Å. (2008). Functional role of unmyelinated tactile afferents in human hairy skin: Sympathetic response and perceptual localization. Experimental Brain Research, 184(1), 135-140. http://doi.org/10.1007/s00221-007-1175-x

Olausson, H., Wessberg, J., Morrison, I., McGlone, F., \& Vallbo, Å. (2010). The neurophysiology of unmyelinated tactile afferents. Neuroscience and Biobehavioral Reviews, 34(2), 185-191. 
http://doi.org/10.1016/j.neubiorev.2008.09.011

Pawling, R., Cannon, P. R., McGlone, F. P., \& Walker, S. C. (2017). C-tactile afferent stimulating touch carries a positive affective value. PLOS ONE, 12(3), 1-15. http://doi.org/10.1371/journal.pone.0173457

Perini, I., Morrison, I., \& Olausson, H. (2015). Seeking pleasant touch: neural correlates of behavioral preferences for skin stroking. Frontiers in Behavioral Neuroscience, 9(February), 1-9. http://doi.org/10.3389/fnbeh.2015.00008

Petrovic, P., Kalisch, R., Singer, T., \& Dolan, R. J. (2008). Oxytocin Attenuates Affective Evaluations of Conditioned Faces and Amygdala Activity. Journal of Neuroscience, 28(26), 6607-6615. http://doi.org/10.1523/JNEUROSCI.457207.2008

Pierce, J. W. (2009). Generating stimuli for neuroscience using PsychoPy. Front. Neuroinform, 2(10). http://doi.org/doi:10.3389/neuro.11.010.2008

Pool, E., Brosch, T., Delplanque, S., \& Sander, D. (2016). Attentional bias for positive emotional stimuli : A meta-analytic investigation. Psychological Bulletin, 142(1), 79-106. http://doi.org/10.1037/bul0000026

Schirmer, A., Teh, K. S., Wang, S., Vijayakumar, R., Ching, A., Nithianantham, D., ... Cheok, A. D. (2011). Squeeze me, but don't tease me: human and mechanical touch enhance visual attention and emotion discrimination. Social Neuroscience, 6(3), 219-30. http://doi.org/10.1080/17470919.2010.507958

Schneider, W., Eschman, A., \& Zuccolotto, A. (2012). E-Prime Reference Guide. Pittsburg: Psychology Software Tools, Inc.

Spapé, M. M., Harjunen, V., \& Ravaja, N. (2017). Effects of touch on emotional face processing: A study of event-related potentials, facial EMG and cardiac activity. 
Biological Psychology, 124, 1-10. http://doi.org/10.1016/j.biopsycho.2017.01.002

Suvilehto, J. T., Glerean, E., Dunbar, R. I. M., Hari, R., \& Nummenmaa, L. (2015).

Correction for Suvilehto et al., Topography of social touching depends on emotional bonds between humans. Proceedings of the National Academy of Sciences, 112(48), E6718-E6718. http://doi.org/10.1073/pnas.1521810112

Triscoli, C., Ackerley, R., \& Sailer, U. (2014). Touch satiety: differential effects of stroking velocity on liking and wanting touch over repetitions. PloS One, 9(11), e113425. http://doi.org/10.1371/journal.pone.0113425

Trotter, P. D., McGlone, F., McKie, S., McFarquhar, M., Elliott, R., Walker, S. C., \& Deakin, J. F. W. (2016). Effects of acute tryptophan depletion on central processing of CT-targeted and discriminatory touch in humans. European Journal of Neuroscience, 1-12. http://doi.org/10.1111/ejn.13298

Vallbo, a B., Olausson, H., \& Wessberg, J. (1999). Unmyelinated afferents constitute a second system coding tactile stimuli of the human hairy skin. Journal of Neurophysiology, 81(6), 2753-2763.

Voos, A. C., Pelphrey, K. a., \& Kaiser, M. D. (2013). Autistic traits are associated with diminished neural response to affective touch. Social Cognitive and Affective Neuroscience, 8(4), 378-386. http://doi.org/10.1093/scan/nss009

Vrontou, S., Wong, A. M., Rau, K. K., Koerber, H. R., \& Anderson, D. J. (2013). Genetic identification of $\mathrm{C}$ fibres that detect massage-like stroking of hairy skin in vivo. Nature, 493(7434), 669-73. http://doi.org/10.1038/nature11810

Walker, S. C., \& McGlone, F. P. (2013). The social brain: Neurobiological basis of affiliative behaviours and psychological well-being. Neuropeptides, 47(6), 379393. http://doi.org/10.1016/j.npep.2013.10.008 
Watson, D., Clark, L. A., \& Tellegen, A. (1988). Development and validation of brief measures of positive and negative affect: the PANAS scales. Journal of Personality and Social Psychology, 54(6), 1063. 